\title{
Energy Efficiency Maximization through Cooperative Transmit and Receive Antenna Selection for Multicell MU-MIMO System
}

\author{
Yanjie Dong, ${ }^{1,2}$ Yinghai Zhang, ${ }^{1}$ Weidong Wang, ${ }^{1}$ Gaofeng Cui, ${ }^{1}$ and Yang Yu ${ }^{1}$ \\ ${ }^{1}$ Key Laboratory of Universal Wireless Communication of Ministry of Education, Beijing University of Posts and Telecommunications, \\ Beijing 100876, China \\ ${ }^{2}$ China Mobile Design Institute, Beijing 100876, China
}

Correspondence should be addressed to Yanjie Dong; dongyanjie@cmdi.chinamobile.com

Received 10 January 2013; Accepted 9 April 2013

Academic Editor: Mugen Peng

Copyright (C) 2013 Yanjie Dong et al. This is an open access article distributed under the Creative Commons Attribution License, which permits unrestricted use, distribution, and reproduction in any medium, provided the original work is properly cited.

\begin{abstract}
The capacity of Multiple Input Multiple Output (MIMO) system is highly related to the number of active antennas. But as the active antenna number increases, the MIMO system will consume more energy. To maximize the energy efficiency of MIMO system, we propose an antenna selection scheme which can maximize the energy efficiency of BS cluster. In the scheme, ergodic energy efficiency is derived according to large scale channel state information (CSI). Based on this ergodic energy efficiency, we introduce a cost function varied with the number of antennas, in which the effect to the energy efficiency of both the serving BS and the neighbor BS is considered. With this function, we can transform the whole system optimization problem to a sectional optimization problem and obtain a suboptimal antenna set using a heuristic algorithm. Simulation results verify that the proposed approach performs better than the comparison schemes in terms of network energy efficiency and achieves $98 \%$ network energy efficiency of the centralized antenna selection scheme. Besides, since the proposed scheme does not need the complete CSI of the neighbor BS, it can effectively reduce the signaling overhead.
\end{abstract}

\section{Introduction}

Due to the challenge of global climate change, reducing carbon dioxide emissions is of high priority for industries. In wireless communications, "green communication" is highly appreciated by many research bodies, which aims to improve the network energy efficiency [1]. Researchers proposed a lot of energy-saving methods on different levels of the wireless network, such as application of new power source, development of lower power consumption device, improvement of network framework, and energy-saving mode of network node [2]. Base station (BS) is one of the most important nodes in wireless network, and it consumes $60-80$ percent [2] of the whole network's power. Thus, how to improve the energy efficiency of the BS is the key to realize energy saving of the wireless network.

The existing techniques about BS energy saving can be divided into two categories. One is based on OFDMA [3], and the other is based on Multiple Input Multiple Output (MIMO). For the former, one carrier can only be allocated to one single user, while it can be allocated to multiple users for the latter. We also call it as "MU-MIMO" when multiple users use the same carrier. MU-MIMO technology can improve the system capacity without increasing the system power consumption and carrier bandwidth. So MUMIMO is regarded as one of the key technologies of IMTAdvanced by 3GPP [4-6] and IEEE $802.16 \mathrm{~m}$. In this paper, we focus on the energy efficiency of MU-MIMO systems.

According to the definition of energy efficiency [7], it is mainly restricted by two factors in MU-MIMO system. One is the system capacity; the other is the system power consumption. The capacity of MU-MIMO system is affected by many factors, such as the distance between user and BS, the number of data stream, antenna beam-forming gain, and number of users. For the single cell MU-MIMO system, the system capacity grows as the number of transmit antenna increases. But in multiple cell system, it is not always the truth because of the interference caused by transmit antennas of the neighbor cells. Besides, the system power consumption increases with the growth of transmit antennas. This paper 
focuses on how to maximize the system energy efficiency by selecting the transmitting antennas and the serving users in multiple cell systems.

1.1. Related Work. MIMO system can effectively improve link quality or increase system capacity. The performance of polarized MIMO is investigated in [8]. However, the deployment of MIMO system requires a high digital signal processing (DSP) ability of the BS which is equipped with a mass of radio circuit. In order to achieve the gains of MIMO system in an economic and less complex way, the application of antenna selection technique has attracted significant attention in recent years. The work in $[9,10]$ analyzes the impact of antenna selection in MIMO system. In [11, 12], lowcomplexity transmit antenna selection is investigated, and [11] proposes an inverse matrix update method which greatly reduces the matrix operation complexity of transmit antenna selection. In [13-15], receive antenna selection schemes are investigated when the number of transmit antenna is fixed. The work in [13] proposes a low-complexity scheme based on the capacity/channel norm. In order to improve system capacity further, the work in [16] proposes a joint transmit and receive antenna selection strategy. In [17], the performance of transmit and receive antenna selection based on bit error rate in flat fading channel is analyzed. However, these schemes mentioned previously are based on single BS scenario without considering the influence of interference from neighbor BSs. Reference [18] uses statistical information of neighbor BSs on transmit antenna set selection to avoid vast signaling exchanges among neighbor BSs. In [19], a coordinated beamforming method is given for downlink multicell MIMO network. However, all of these previous researches are based on the assumption that the BS can use all the available radio circuit to maximize system capacity or minimize link error probability. This assumption does not consider the power consumption and cannot ensure the maximization of the BS energy efficiency.

In [20], taking into account both the transmit power and power consumed by circuits, energy efficiency problem of MIMO system based on short range communication (SRD) is investigated. The authors in [20] show that the energy efficiency of MIMO system would be greater than that of SISO system only if a combination of adaptive modulation techniques and MIMO techniques is used. The work in [21] analyzes the impact of the spatial diversity on energy efficiency and concludes that antenna selection is in general the most energy efficient option for MIMO systems. In [22], a power amplifier selection scheme is given to improve the energy efficiency. The work in [23] analyzes the impact of antenna selection on improving the energy efficiency. In [24], system energy efficiency is improved through antenna selection for downlink broadcast channel. The impact of transmit antenna selection on energy efficiency in distributed antenna systems (DASs) is analyzed in [25]. The work in [26] investigates the problem of user selection and power allocation to maximize energy efficiency. In [27], energy efficiency is maximized through joint optimization of transmit power, number of transmit antennas, and transmit/receive antenna sets. However, these researches mentioned previously are all based on single-cell scenario and do not consider the impact of the number of transmit antennas and the active receive antenna set on the energy efficiency. The work in [28] proposes an energy efficient coordinated beamforming method for clustered MIMO network. The work in [29] maximizes network energy efficiency through BS transmit antenna selection and transmission mode selection. However, since this method is based on interference nulls and uses ergodic search strategy and considers the three-cell singleuser scenario, the computation complexity will be very high when the number of BSs increases, and it cannot be applied to multiuser scenario.

1.2. Contribution. In this paper, we focus on transmit and receive antenna selection for multicell MU-MIMO system to maximize the system energy efficiency. The main contribution of our work can be summarized as follows.

1.2.1. Ergodic Energy Efficiency Based on Large Scale CSI. We derive the available ergodic energy efficiency obtained by BS. This ergodic energy efficiency is only related to the number of BS transmit antenna and large scale CSI between the UE and its severing BS.

\subsubsection{Transmit and Receive Antenna Selection Scheme Based} on Maximizing the Energy Efficiency of BS Cluster. Based on the ergodic energy efficiency, we introduce a cost function of transmit and receive antenna selection. This cost function consists of two parts: one part is ergodic energy efficiency of the target BS; the other is energy efficiency change of neighbor BSs after the number of transmit antennas of the target BS changes. With this function, we can convert the global optimization of overall system energy efficiency to local optimization problem based on the maximization of BS cluster energy efficiency. Then we develop a heuristic transmit and receive antenna selection algorithm to solve the problem. Since ergodic energy efficiency is only related to the number of BS transmit antennas and large scale CSI between the UE and its severing BS, the proposed scheme can effectively reduce the signaling overhead among different BSs.

1.3. Organization. The remainder of this paper is organized as follows. Section 2 describes the system model. Section 3 derives ergodic energy efficiency and develops a heuristic transmit and receive antenna selection scheme based on this term. Section 4 gives simulation results and analysis. Finally, conclusions are drawn in Section 5.

In this paper, $\mathbf{A}^{H},|\mathbf{A}|,\|\mathbf{A}\|_{F}^{2}$, and $\mathbf{A}^{-1}$ express the Hermitian transpose, determinant, Frobenius norm, and inverse of matrix A, respectively. The expectation operator is denoted by $E\{\cdot\}$.

\section{System Model}

In this paper, we consider a downlink multicell MU-MIMO system model which consists of a cellular network with $J$ cells; each cell consists of $K_{i}$ users. We assume that each cell has 
one BS consisting of $N_{\max }$ transmit antennas, and each user is equipped with single antenna. Although $N_{\max }$ is assumed to be the same for different BSs, it can vary according to the type of individual BS. And each user can only be served by one BS. Partial CSI is shared among BSs; that is, BS can obtain the CSI between itself and its serving users and users it interferes with. The received signal of user $k$ can be expressed as

$$
\begin{aligned}
& y_{k, i}= \sqrt{\frac{\rho_{k, i}}{M_{i}}} \mathbf{H}_{k, i} \mathbf{W}_{k, i} \mathbf{x}_{k, i}+\sqrt{\frac{\rho_{k, i}}{M_{i}}} \sum_{k \neq l}^{K_{i}} \mathbf{H}_{k, i} \mathbf{W}_{l, i} \mathbf{x}_{l, i} \\
&+\sum_{i \neq j, j=1}^{J} \sum_{k=1}^{K_{j}} \sqrt{\frac{\rho_{k, j}}{M_{j}}} \mathbf{H}_{k, j} \mathbf{W}_{\bar{k}, j} \mathbf{x}_{\bar{k}, j}+\mathbf{n}_{k, i},
\end{aligned}
$$

where $\rho_{k, i}=P / L_{k, i}, L_{k, i}$ is the path loss between user $k$ and $\mathrm{BS} i, P$ is the overall BS transmit power, $M_{i}$ is the number of transmit data streams of $\mathrm{BS} i$, which can be different according to $i$, and $\mathbf{x}_{k, i}$ is the transmit signal of user $k$. We assume that different data streams adopt the same transmit power; that is, $E\left\{\mathbf{x}_{k, i} \mathbf{x}_{k, i}^{\mathbf{H}}\right\}=1 . \mathbf{H}_{k, i}$ denotes the small scale fading between user $k$ and $\mathrm{BS} i$, the fading coefficients in $\mathbf{H}_{k, i}$ are i.i.d. with zero-mean and variance $1, \mathbf{W}_{k, i} \in C^{N_{i} \times 1}$ is the precoding matrix of user $k,\left\|\mathbf{W}_{k, i}\right\|_{F}^{2}=1, N_{i}\left(N_{i} \leq N_{\max }\right)$ is the transmit antenna activated by $\mathrm{BS} i$, and $\mathbf{n}_{k, i}$ is additive white Gaussian noise (AWGN) with zero-mean and variance 1 . The second term on the right side of the equal sign in formula (1) is interuser interference (IUI), and the third term is intercell interference (ICI).

Assuming that Zero Forcing Beamforming (ZFBF) [30] is adopted to eliminate IUI, the direction of user $k$ 's precoding vectors must be the same as the projections of $\mathbf{H}_{k, i}$ on the null space of $\overline{\mathbf{H}}=\left[\mathbf{H}_{1, i}^{\mathbf{H}}, \mathbf{H}_{2, i}^{\mathbf{H}}, \mathbf{H}_{k-1, i}^{\mathbf{H}}, \mathbf{H}_{k+1, i}^{\mathbf{H}}, \mathbf{H}_{K_{i}, i}^{\mathbf{H}}\right]^{\mathbf{H}}$. Thus, the normalized $\mathbf{W}_{k, i}$ can be expressed as

$$
\mathbf{W}_{k, i}=\left(\mathbf{I}-\overline{\mathbf{H}}^{\mathbf{H}}\left(\overline{\mathbf{H}} \overline{\mathbf{H}}^{\mathbf{H}}\right)^{-1} \overline{\mathbf{H}}\right) \mathbf{H}_{k, i}
$$

where, $\mathbf{I} \in C^{N_{i} \times N_{i}}$ is an identity matrix.

After the IUI has been eliminated, (1) can be rewritten as

$$
\begin{aligned}
y_{k, i}= & \sqrt{\frac{\rho_{k, i}}{M_{i}}} \mathbf{H}_{k, i} \mathbf{W}_{k, i} \mathbf{x}_{k, i} \\
& +\sum_{i \neq j, j=1}^{J} \sum_{\bar{k}=1}^{K_{j}} \sqrt{\frac{\rho_{k, j}}{M_{j}}} \mathbf{H}_{k, j} \mathbf{W}_{\bar{k}, j} \mathbf{x}_{\bar{k}, j}+\mathbf{n}_{k, i} .
\end{aligned}
$$

Therefore, the received signal to interference plus noise (SINR) can be expressed as

$$
\operatorname{SINR}_{k, i}=\frac{\left(\rho_{k, i} / M_{i}\right) \mathbf{H}_{k, i} \mathbf{W}_{k, i} \mathbf{W}_{k, i}^{\mathbf{H}} \mathbf{H}_{k, i}^{\mathbf{H}}}{1+\sum_{j=1, i \neq j}^{J} \sum_{\bar{k}=1}^{K_{j}}\left(\rho_{k, j} / M_{j}\right) \mathbf{H}_{k, j} \mathbf{W}_{\bar{k}, j} \mathbf{W}_{\bar{k}, j}^{\mathbf{H}} \mathbf{H}_{k, j}^{\mathbf{H}}} .
$$

The ergodic capacity of single user can be expressed as

$$
C_{k, i}=E\left[B \log _{2}\left(1+\operatorname{SINR}_{k, i}\right)\right] .
$$

Thus, the system ergodic capacity for $J$ BSs can be obtained as

$$
C=\sum_{i=1}^{J} \sum_{k=1}^{M_{i}} C_{k, i}
$$

Furthermore, the power consumption of BSs can be formulated as [7]

$$
P_{\text {total }, i}\left(N_{i}, P, B\right)=\frac{P}{\eta}+P_{D}+P_{S},
$$

where $P$ is the transmit power of BS, $\eta$ is the amplifier efficiency, $P_{D}$ is the dynamic power of $\mathrm{BS}$, which is determined by the number of transmit antennas and bandwidth, $P_{D}=$ $N_{i} P_{\text {cir }}+B P_{\text {ac }}+N_{i} P_{\text {sp,bw }} B, P_{\text {cir }}$ is the power consumed in circuits, $P_{\mathrm{ac}}$ is the power consumed by A/D converter, $P_{\mathrm{sp}, \mathrm{bw}}$ is the power consumed by the signal processing unit, $B$ is the system bandwidth, and $P_{S}$ is the static power of BS which consists of power consumption such as air-condition and power supply. [29]

The network energy efficiency (NEE) can be defined as

$$
\mathrm{NEE}=\frac{\sum_{i=1}^{J} \sum_{k=1}^{M_{i}} C_{k, i}}{\sum_{i=1}^{J} P_{\text {total }, i}\left(N_{i}, P, B\right)} .
$$

The maximization problem of system NEE can be expressed as

$$
\begin{gathered}
\max _{\substack{\left\{N_{i}\right\}\left\{M_{i}\right\} \\
\Omega_{T, i}, \Omega_{R, i}}}\left\{\frac{\sum_{i=1}^{J} \sum_{k=1}^{M_{i}} C_{k, i}}{\sum_{i=1}^{J} P_{\text {total }, i}\left(N_{i}, P, B\right)}\right\} \\
\text { subject to }\left\{\begin{array}{l}
1 \leq N_{i} \leq N_{\max } \\
1 \leq M_{i} \leq N_{i} \\
\Omega_{T, i} \subseteq \Phi_{T, i} \\
\Omega_{R, i} \subseteq \Phi_{R, i},
\end{array}\right.
\end{gathered}
$$

where $\Omega_{T, i}$ denotes the set of transmit antennas activated by BSi, $\Phi_{T, i}$ is the transmit antenna set of BSi, $\Omega_{R, i}$ is the set of activated receive antennas of users served by $\mathrm{BS} i$, and $\Phi_{R, i}$ is the receive antenna set of users served by BSi.

\section{Transmit and Receive Antenna Selection Scheme Based on Maximizing the Energy Efficiency of BS Cluster}

From (9), we can find that NEE relates to the capacity and power consumption of all the BSs in the network. Especially for the available capacity, it is determined by not only its selection of transmit and receive antenna sets, but also its neighbor BSs' selection of transmit and receive antenna sets. The latter influences the interference suffered by its serving users. Therefore, maximization of NEE is a nonconvex problem. In order to resolve this problem, we first derive ergodic energy efficiency based on large scale CSI. Then a transmit and receive antenna selection strategy based on cost function is proposed to leverage this ergodic energy efficiency. 
3.1. Ergodic Energy Efficiency Based on Large Scale CSI. Since each user is equipped with single antenna and $\mathbf{H}_{k, i} \mathbf{W}_{k, i} \mathbf{W}_{k, i}^{\mathbf{H}} \mathbf{H}_{k, i}^{\mathbf{H}}=\left\|\mathbf{H}_{k, i} \mathbf{W}_{k, i}\right\|_{F}^{2}$ in (4), $\operatorname{SINR}_{k, i}$ can be rewritten as

$$
\begin{aligned}
\operatorname{SINR}_{k, i} & =\frac{\left(\rho_{k, i} / M_{i}\right)\left\|\mathbf{H}_{k, i} \mathbf{W}_{k, i}\right\|_{F}^{2}}{1+\sum_{j=1, i \neq j}^{J} \sum_{\bar{k}=1}^{M_{j}}\left(\rho_{k, j} / M_{j}\right)\left\|\mathbf{H}_{k, j} \mathbf{W}_{\bar{k}, j}\right\|_{F}^{2}} \\
& =\frac{\left(\rho_{k, i} / M_{i}\right)\left\|\mathbf{H}_{k, i} \mathbf{W}_{k, i}\right\|_{F}^{2}}{1+\sum_{j=1, i \neq j}^{J}\left(\rho_{k, j} / M_{j}\right) \sum_{\bar{k}=1}^{M_{j}}\left\|\mathbf{H}_{k, j} \mathbf{W}_{\bar{k}, j}\right\|_{F}^{2}} .
\end{aligned}
$$

In (10), because $\mathbf{W}_{k, i}$ belongs to the null space of the channel set of the other serving users in the same cell, $\left\|\mathbf{H}_{k, i} \mathbf{W}_{k, i}\right\|_{F}^{2}$ obeys Chi-square distribution with degree of freedom $2\left(N_{i}-\left(M_{i}-1\right)\right)$, that is, $\left\|\mathbf{H}_{k, i} \mathbf{W}_{k, i}\right\|_{F}^{2} \sim \chi_{2\left(N_{i}-\left(M_{i}-1\right)\right)}^{2}$, where $M_{i}$ is the number of data streams. Since $\mathbf{W}_{\bar{k}, j}$ is uncorrelated with $\mathbf{H}_{k, j},\left\|\mathbf{H}_{k, j} \mathbf{W}_{\bar{k}, j}\right\|_{F}^{2} \sim \chi_{2}^{2}$ is independent with the number of data streams. From the above mentioned, when the BS adopts ZFBF to implement beamforming, useful signal strength can be regarded as random variable which obeys Chi-square distribution with degree of freedom $2\left(N_{i}-\right.$ $\left.\left(M_{i}-1\right)\right)$, and the signal strength of interferences from the neighbor cells can be regarded as random variable which obeys Chi-square distribution with degree of freedom 2. Defining $Z_{k, i}=\left\|\mathbf{H}_{k, i} \mathbf{W}_{k, i}\right\|_{F}^{2}$ and $Y_{k, j, \bar{k}}=\left\|\mathbf{H}_{k, j} \mathbf{W}_{\bar{k}, j}\right\|_{F}^{2}$, where $Z_{k, i}$ and $Y_{k, j, \bar{k}}$ are mutually independent, $Z_{k, i} \sim \chi_{2\left(N_{i}-\left(M_{i}-1\right)\right)}^{2}$ and $Y_{k, j, \bar{k}} \sim \chi_{2}^{2},(10)$ can be rewritten as

$$
\begin{aligned}
\operatorname{SINR}_{k, i} & =\frac{\left(\rho_{k, i} / M_{i}\right) Z_{k, i}}{1+\sum_{j=1, i \neq j}^{J}\left(\rho_{k, j} / M_{j}\right) \sum_{\bar{k}=1}^{M_{j}} Y_{k, j, \bar{k}}} \\
& \leq \frac{\left(\rho_{k, i} / M_{i}\right) Z_{k, i}}{1+\sum_{j=1, i \neq j}^{J}\left(\rho_{k, j} / N_{\text {max }}\right) Y_{k, j, \bar{k}}} .
\end{aligned}
$$

When users suffer weaker interference from neighbor cells, the upper bound of $\operatorname{SINR}_{k, i}$ in (11) is tight. Defining $\alpha_{k, i}=$ $\rho_{k, i} / M_{i}, \beta_{k, j}=\rho_{k, j} / N_{\max }$ and $Y_{k, j}=Y_{k, j, \bar{k}}, X_{k, i}$ can be expressed as

$$
X_{k, i}=\frac{\alpha_{k, i} Z_{k, i}}{1+\sum_{j=1, i \neq j}^{J} \beta_{k, j} Y_{k, j}}
$$

Thus, the ergodic capacity of single user can be expressed as

$$
\begin{aligned}
C_{k, i}\left(\alpha_{k, i}, \beta_{k, j}, N_{i}, M_{i}, B\right) & \leq E\left[B \log _{2}\left(1+X_{k, i}\right)\right] \\
& =E\left[B \log _{2}(e) \ln \left(1+X_{k, i}\right)\right] \\
& =B \log _{2}(e) E\left[\ln \left(1+X_{k, i}\right)\right] .
\end{aligned}
$$

Then, the upper bound of network capacity can be expressed as

$$
\bar{C}_{k, i}\left(\alpha_{k, i}, \beta_{k, j}, N_{i}, M_{i}, B\right)=B \log _{2}(e) E\left[\ln \left(1+X_{k, i}\right)\right] .
$$

Theorem 1. (1) When $J=1$,

$$
\begin{aligned}
& \bar{C}_{k, i}\left(\alpha_{k, i}, \beta_{k, j}, N_{i}, M_{i}, B\right) \\
& \quad=B \log _{2}(e) \sum_{r=0}^{N_{i}-M_{i}} \sum_{l=0}^{r} \frac{\left(\alpha_{k, i}\right)^{l+1-r}}{\beta_{k, 1}(r-l) !} I_{1}\left(\frac{1}{\alpha_{k, i}}, \frac{\alpha_{k, i}}{\beta_{k, 1}}, r, l+1\right) .
\end{aligned}
$$

(2) When $J=2$,

$$
\begin{aligned}
\bar{C}_{k, i} & \left(\alpha_{k, i}, \beta_{k, j}, N_{i}, M_{i}, B\right) \\
= & B \log _{2}(e) \sum_{r=0}^{N_{i}-M_{i}} \sum_{l=0}^{r} \frac{\left(\alpha_{k, i}\right)^{l+1-r}}{\left(\beta_{k, 1}-\beta_{k, 2}\right)(r-l) !} \\
& \times\left[I_{1}\left(\frac{1}{\alpha_{k, i}}, \frac{\alpha_{k, i}}{\beta_{k, 1}}, r, l+1\right)-I_{1}\left(\frac{1}{\alpha_{k, i}}, \frac{\alpha_{k, i}}{\beta_{k, 2}}, r, l+1\right)\right],
\end{aligned}
$$

where $I_{1}(a, b, c, d)$ is an integral function [31].

Proof. The proof procedure can be found in [31].

Theorem 2. When $J=3$,

$$
\begin{aligned}
\bar{C}_{k, i}\left(\alpha_{k, i}, \beta_{k, j}, N_{i}, M_{i}, B\right) \\
=B \log _{2}(e) \sum_{r=0}^{N_{i}-M_{i}} \sum_{l=0}^{r} \frac{\left(\alpha_{k, i}\right)^{l+1-r}}{(r-l) !} \\
\times\left[\begin{array}{l}
\frac{\beta_{k, 1}}{\left(\beta_{k, 1}-\beta_{k, 2}\right)\left(\beta_{k, 1}-\beta_{k, 3}\right)} I_{1}\left(\frac{1}{\beta_{k, i}}, \frac{\alpha_{k, i}}{\beta_{k, 1}}, r, l+1\right) \\
-\frac{1}{\left(\beta_{k, 1}-\beta_{k, 2}\right)\left(\beta_{k, 2}-\beta_{k, 3}\right)} I_{1}\left(\frac{\alpha_{k, i}}{\alpha_{k, i}}, r, l+1\right) \\
+\frac{\beta_{k, 3}}{\left(\beta_{k, 1}-\beta_{k, 3}\right)\left(\beta_{k, 2}-\beta_{k, 3}\right)} I_{1}\left(\frac{1}{\alpha_{k, i}}, \frac{\alpha_{k, i}}{\beta_{k, 3}}, r, l+1\right)
\end{array}\right] .
\end{aligned}
$$

Proof. For the proof, see the Appendix.

Considering the signal attenuation caused by distance, the users are mainly affected by the interference from the three neighbor BSs. Therefore, this paper only calculates the interference from the three neighbor BSs to the users. For $J>3$ cases, the upper bound of network capacity can be obtained by the same previous method. From (14), the upper bound of network energy efficiency is

$$
\begin{aligned}
\mathrm{NEE}_{\text {upper }}\left(N_{i}, M_{i}, B, P\right) \\
=\frac{\sum_{i=1}^{J} \sum_{k=1}^{M_{i}} \bar{C}_{k, i}\left(\alpha_{k, i}, \beta_{k, j}, N_{i}, M_{i}, B\right)}{\sum_{i=1}^{J} P_{\text {total }, i}\left(N_{i}, P, B\right)} .
\end{aligned}
$$

According to the upper bound of network energy efficiency in (18), $\mathrm{NEE}_{\text {upper }}$ is only related to the large scale CSI between BS and its serving users, the number of transmit antennas, and transmit power. Using (18) to implement antenna selection can effectively reduce the signaling overhead caused by the complete CSI sharing among different BSs. 
3.2. The Introduction of the Cost Function and the Transmit and Receive Antenna Selection Strategy. Based on (18), we can convert the maximization problem of network energy efficiency in (9) into the maximization problem of the upper bound $\mathrm{NEE}_{\text {upper }}$ of network energy efficiency. Then, (9) can be rewritten as

$$
\begin{aligned}
& \max _{\substack{\left\{N_{i}\right\}\left\{M_{i}\right\} \\
\Omega_{T, i}, \Omega_{R, i}}}\left\{\frac{\sum_{i=1}^{J} \sum_{k=1}^{M_{i}} \bar{C}_{k, i}\left(\alpha_{k, i}, \beta_{k, j}, N_{i}, M_{i}, B\right)}{\sum_{i=1}^{J} P_{\text {total }, i}\left(N_{i}, P, B\right)}\right\} \\
& \text { subject to }\left\{\begin{array}{l}
1 \leq N_{i} \leq N_{\max } \\
1 \leq M_{i} \leq N_{i} \\
\Omega_{T, i} \subseteq \Phi_{T, i} \\
\Omega_{R, i} \subseteq \Phi_{R, i} .
\end{array}\right.
\end{aligned}
$$

It can be drawn from (19) that the average network energy efficiency is mainly related to the transmit and receive antenna sets of each BS, when both the bandwidth and the BS transmit power are fixed. However, before one BS determines the transmit antenna set and serving users of itself, it must know the number of transmit antennas in neighbor cells. To ensure the maximization of network energy efficiency, it is necessary to deploy a central processing unit which can select the transmit antennas and serving users of all the BSs. This problem can be resolved by brute-force complete search, but it will become very complex as the number of transmit antennas and serving users increases. Besides, users' CSI must be shared among all the BSs, which will produce lots of signaling overhead.

In this part, we will take the network energy efficiency difference as cost function. The difference is brought by the change of BS transmit antenna number and data stream number. Assuming that the BS bandwidth and transmit power are fixed, the network energy efficiency is only related to the number of BS transmit antennas and data streams. Then (18) can be expressed as

$$
\begin{aligned}
\operatorname{NEE}_{\text {upper }}\left(N_{i}, M_{i}\right)= & \frac{\sum_{i=1}^{J} \sum_{k=1}^{M_{i}} \bar{C}_{k, i}\left(\alpha_{k, i}, \beta_{k, j}, N_{i}, M_{i}\right)}{\sum_{i=1}^{J} P_{\text {total }, i}\left(N_{i}\right)} \\
= & \frac{\sum_{k=1}^{M_{i}} \bar{C}_{k, i}\left(\alpha_{k, i}, \beta_{k, j}, N_{i}, M_{i}\right)}{\sum_{i=1}^{J} P_{\text {total }, i}\left(N_{i}\right)} \\
& +\frac{\sum_{j=1, j \neq i}^{J} \sum_{\bar{k}=1}^{M_{j}} \bar{C}_{\bar{k}, i}\left(\alpha_{\bar{k}, i}, \beta_{\bar{k}, j}, N_{j}, M_{j}\right)}{\sum_{i=1}^{J} P_{\text {total }, i}\left(N_{i}\right)} .
\end{aligned}
$$

When the number of BSi' transmit antennas changes from $N_{i}$ to $N_{i}+n$, and the number of BSi data streams changes from $M_{i}$ to $M_{i}+m$, while the number of other BS's transmit antennas and data streams stays the same, the network energy efficiency can be expressed as

$$
\begin{aligned}
\mathrm{NEE}_{\text {upper }}\left(N_{i}+n, M_{i}+m\right) & \\
= & \frac{\sum_{i=1}^{J} \sum_{k=1}^{M_{i}} \bar{C}_{k, i}\left(\alpha_{k, i}, \beta_{k, j}, N_{i}+n, M_{i}+m\right)}{\sum_{i=1}^{J} P_{\text {total }, i}\left(N_{i}\right)} \\
= & \frac{\sum_{k=1}^{M_{i}} \bar{C}_{k, i}\left(\alpha_{k, i}, \beta_{k, j}, N_{i}+n, M_{i}+m\right)}{\sum_{j \neq i}^{J} P_{\text {total }, i}\left(N_{j}\right)+P_{\text {total }, i}\left(N_{i}+n\right)} \\
& +\frac{\sum_{j=1, j \neq i}^{J} \sum_{\bar{k}=1}^{M_{j}} \bar{C}_{\bar{k}, i}\left(\alpha_{\bar{k}, i}, \beta_{\bar{k}, j}, N_{j}, M_{j}\right)}{\sum_{j \neq i}^{J} P_{\text {total }, i}\left(N_{j}\right)+P_{\text {total }, i}\left(N_{i}+n\right)} .
\end{aligned}
$$

In (14), the changes of the number of one BS's transmit antennas and data streams will not affect the available capacity $\sum_{\bar{k}=1}^{M_{j}} \bar{C}_{\bar{k}, i}$ that can be gotten by its neighbor BSs. Defining $G=\sum_{j=1, j \neq i}^{J} \sum_{\bar{k}=1}^{M_{j}} \bar{C}_{\bar{k}, i}\left(\alpha_{\bar{k}, i}, \beta_{\bar{k}, j}, N_{j}, M_{j}\right)$, then the network energy efficiency difference brought by the change of transmit antenna can be expressed as

$$
\begin{aligned}
& \mathrm{NEE}_{\text {upper }}\left(N_{i}+n, M_{i}+m\right)-\mathrm{NEE}_{\text {upper }}\left(N_{i}, M_{i}\right) \\
& =\frac{\sum_{k=1}^{M_{i}} \bar{C}_{k, i}\left(\alpha_{k, i}, \beta_{k, j}, N_{i}+n, M_{i}+m\right)}{\sum_{j \neq i}^{J} P_{\text {total }, i}\left(N_{j}\right)+P_{\text {total }, i}\left(N_{i}+n\right)} \\
& +\frac{\sum_{k=1}^{M_{i}} \bar{C}_{k, i}\left(\alpha_{k, i}, \beta_{k, j}, N_{i}, M_{i}\right)}{\sum_{i=1}^{J} P_{\text {total }, i}\left(N_{i}\right)} \\
& +G\left(\frac{1}{\sum_{j \neq i}^{J} P_{\text {total }, i}\left(N_{j}\right)+P_{\text {total }, i}\left(N_{i}+n\right)}\right. \\
& =\mathrm{CEE}_{i}\left(N_{i}+n, M_{i}+m\right)-\mathrm{CEE}_{i}\left(N_{i}, M_{i}\right)+\Delta \mathrm{CEE}_{j} .
\end{aligned}
$$

Defining cost function $S=\operatorname{NEE}_{\text {upper }}\left(N_{i}+n, M_{i}+m\right)-$ $\operatorname{NEE}_{\text {upper }}\left(N_{i}, M_{i}\right)$, we have

$$
\begin{aligned}
S & =\operatorname{NEE}_{\text {upper }}\left(N_{i}+n, M_{i}+m\right)-\operatorname{NEE}_{\text {upper }}\left(N_{i}, M_{i}\right) \\
& =\operatorname{CEE}_{i}\left(N_{i}+n, M_{i}+m\right)-\operatorname{CEE}_{i}\left(N_{i}, M_{i}\right)+\Delta \operatorname{CEE}_{j}(n),
\end{aligned}
$$

where, $\operatorname{CEE}_{i}\left(N_{i}, M_{i}\right)=\sum_{k=1}^{M_{i}} \bar{C}_{k, i}\left(\alpha_{k, i}, \beta_{k, j}, N_{i}, M_{i}\right) /$ $\sum_{i=1}^{J} P_{\text {total }, i}\left(N_{i}\right)$

$$
\begin{aligned}
& \Delta \mathrm{CEE}_{j}(n) \\
&=G\left(\frac{1}{\sum_{j \neq i}^{J} P_{\text {total }, i}\left(N_{j}\right)+P}\right. \\
&\left.-\frac{1}{\sum_{i=1}^{J} P_{\text {total }, i}\left(N_{i}\right)}\right) .
\end{aligned}
$$$$
=G\left(\frac{1}{\sum_{j \neq i}^{J} P_{\text {total }, i}\left(N_{j}\right)+P_{\text {total }, i}\left(N_{i}+n\right)}\right.
$$ 
In (23), $\mathrm{CEE}_{i}\left(N_{i}+n, M_{i}+m\right)-\mathrm{CEE}_{i}\left(N_{i}, M_{i}\right)$ and $\Delta \mathrm{CEE}_{j}(n)$ denote the difference on energy efficiency of target BS itself and the difference on energy efficiency of the other BSs, respectively. We assume that the number of transmit antennas and data streams of neighbor BSs remains unchanged, then $G$ remains unchanged, and $\Delta \operatorname{CEE}_{j}(n)$ is only related to $n$.

Based on these properties of (23) mentioned previously, we develop a transmit and receive antenna selection scheme based on the maximization of BS cluster energy efficiency. In this scheme, a BS cluster consists of seven BSs, and each of them implements transmit and receive antenna selection in turn. In this case, it can ensure that the transmit antenna sets and serving user groups of the six neighbor BSs remain unchanged when each BS implements transmit and receive antenna selection, which will avoid the iteration. Each BS first determines its available number of transmit antennas and receive antenna set using a heuristic algorithm and then activates the antennas which will maximize the upper bound of capacity in the transmit antenna set. After determining the transmit and receive antennas of itself, the target BS shares its information of upper limit capacity with its neighbor BSs. Based on this information, the other BSs can calculate their cost function. The transmit and receive antenna selection scheme of a single BS can be summarized in Algorithm 1.

\section{Simulation Results}

In this section, we present simulation results of our proposed scheme and investigate its performance. In particular, we compare the performance of our scheme with that of single cell CAS, single cell EEAS, and centralized EEAS. For centralized EEAS, each user's complete CSI must be shared among all the BSs, and a central processing unit is needed to implement transmit and receive antenna selection for all the users in the system.

In the simulation, we adopt 7-cell downlink MU-MIMO system model. Each cell uses the same frequency resource. We assume that each BS has $N$ transmit antennas, and each user is equipped with single antenna. Users location obey uniform distribution in the coverage of the BS. The path loss model base on distance is $37.6 \log (d)+128.1$, and the standard deviation of shadowing is $8 \mathrm{~dB}$. According to energy demands for each parts of the BS, we set $\eta=0.38, P_{\text {cir }}=66.4 \mathrm{~W}$, $P_{\mathrm{ac}}=1.82 \mu \mathrm{W} / \mathrm{Hz}, P_{\mathrm{sp}, \mathrm{bw}}=3.32 \mu \mathrm{W} / \mathrm{Hz}, P_{S}=36.4 \mathrm{~W}$, and $B=5 \mathrm{MHz}$.

Figure 1 shows NEE's change as the user number increases. From the figure, the NEE increases with the user number per cell because more multiuser diversity gain is obtained. Since cluster EEAS considers the impact of ICI on NEE, its NEE is much higher than single cell CAS and single cell EEAS. For example, when user number per cell is 16, cluster EEAS can achieve about 13\% and 28\% NEE gain over the single cell CAS and single cell EEAS. In addition, compared with the centralized EEAS, the proposed scheme has only $2 \%$ NEE degradation, but it does not need complete CSI sharing and central processing unit.

Figure 2 shows the NEE versus the cell radius. The simulation keeps the BS transmit power unchanged and

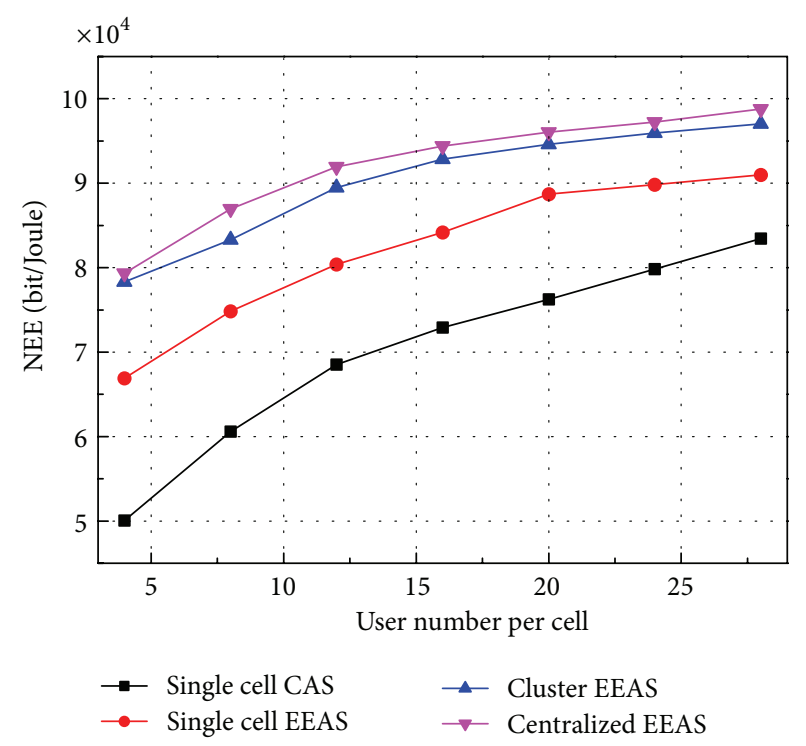

FIgure 1: NEE versus User number per cell with $N_{\max }=4, P=$ $20 \mathrm{~W}$, and cell radius of $1 \mathrm{Km}$.

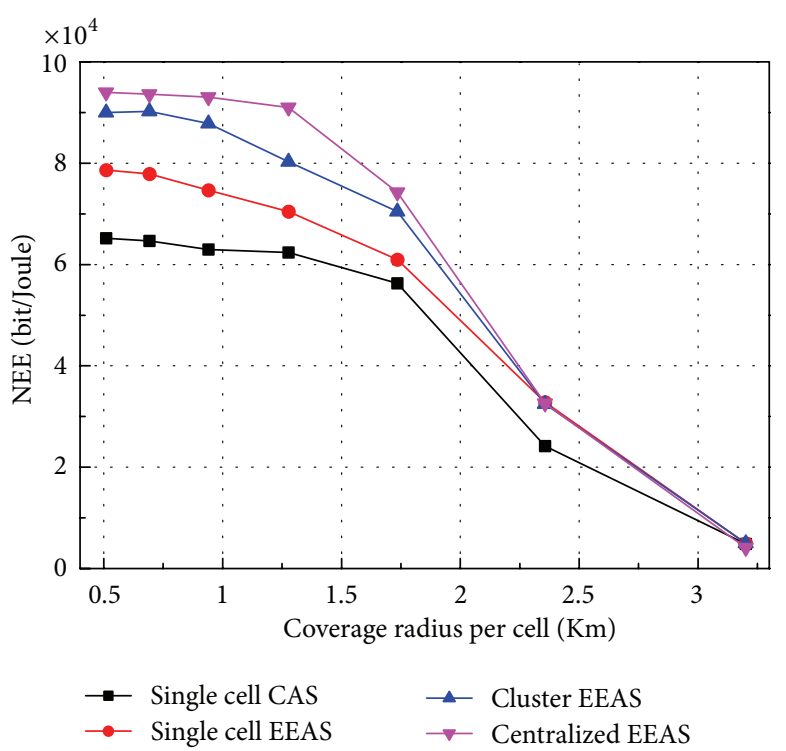

FIgUre 2: NEE versus Coverage radius per cell with $N_{\max }=4, P=$ $20 \mathrm{~W}$, and 10 users per cell.

increases the cell radius from $0.5 \mathrm{Km}$ to $3.5 \mathrm{Km}$. From this figure, we can see that the NEE decreases with the increase of the cell radius, and when the cell radius is greater than $1.5 \mathrm{Km}$, NEE decreases faster. While for the cell radius less than $1.5 \mathrm{Km}$, the capacity decreases less with the increase of the cell radius. This is mainly because that the impact of path loss on SNR is aggravated when the cell radius is large. Besides, when the cell radius is large enough, these four schemes almost obtain the same EEAS. This is mainly because when the cell radius increases, the ICI will recede. On this occasion, the useful signal strength becomes the major influencing factor on NEE, and the gain of cluster EEAS recedes. Thus it can be 


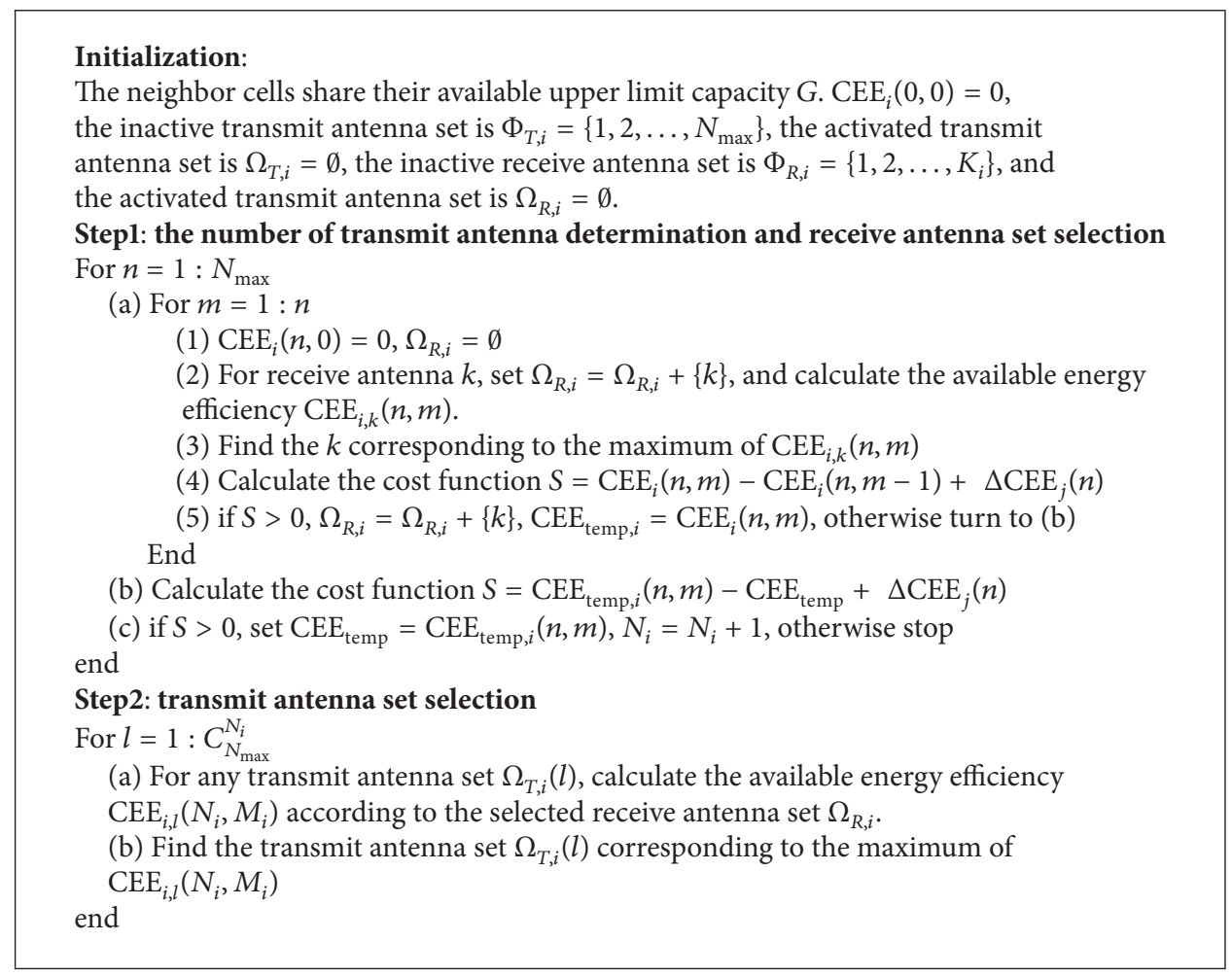

Algorithm 1: Cluster energy efficiency based antenna selection (cluster EEAS).

seen that cluster performs much better in high interference scenario.

The NEE versus the transmit power of BS is shown in Figure 3. On this occasion, there are two main effect factors of NEE: the capacity which can be obtained by the cell and BS transmit power. From this figure, we can see that NEE increases with the growth of the transmit power, but when the BS transmit power is greater than $30 \mathrm{dBm}$, NEE decreases quickly. This proves that the increase of the BS transmit power cannot always bring the growth of BS NEE. Besides, when the BS transmit power is greater than $30 \mathrm{dBm}$, the scheme considering energy efficiency decreases faster than the CAS in NEE. This illustrates that in the schemes considering energy efficiency, the increase of the BS transmit power cannot always bring the increase of cell capacity.

The NEE versus the maximal transmit antenna number of BS is shown in Figure 4. For the CAS of capacity maximum, NEE decreases with the increase of the maximal transmit antenna number. This is mainly because all the transmit antennas have been activated to gain high capacity in this scheme, but more transmit antennas mean more energy consumed by BSs. If the capacity gains brought by activating more transmit antennas cannot compensate the impact of power consumption on NEE, NEE will decrease. In this simulation scenario, activating more transmit antenna goes against the improvement of network NEE obviously. However, for the schemes which consider the NEE, NEE will not decrease with the increase of the maximal transmit antenna number. That is because that the transmit antenna will not be activated when it does not increase NEE in the proposed scheme.

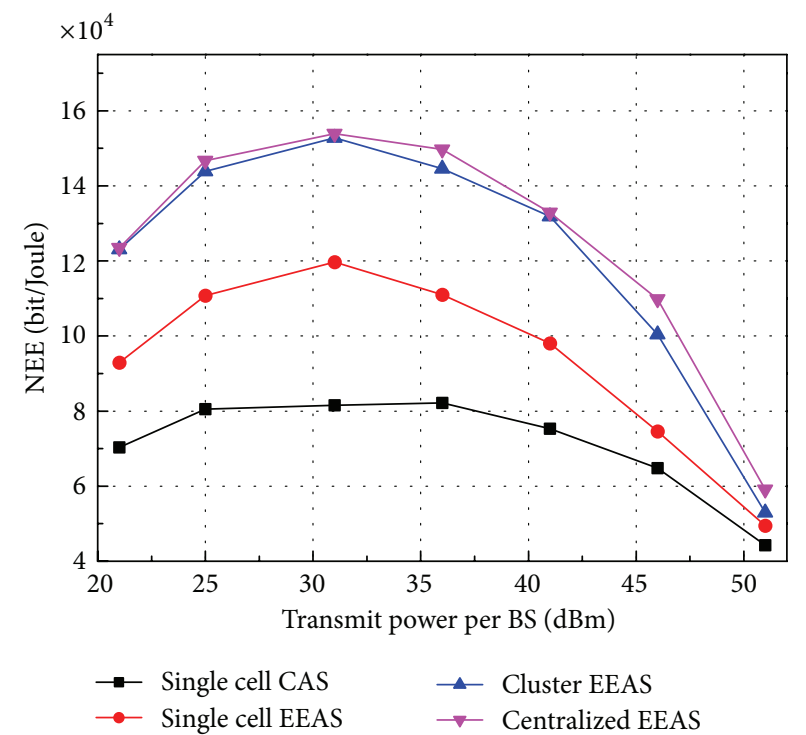

FIgURE 3: NEE versus transmit power per BS with $N_{\max }=4,10$ user per cell and cell radius of $1 \mathrm{Km}$.

Compared with single cell CAS and single cell EEAS, cluster EEAS needs the users to feedback the large scale CSI of interfering channels between users and its interfering BSs. Moreover, a centralized entity is needed to make the BSs in a cluster maximize the energy efficiency in turn. Whereas, centralized EEAS needs a centralized entity to select the 


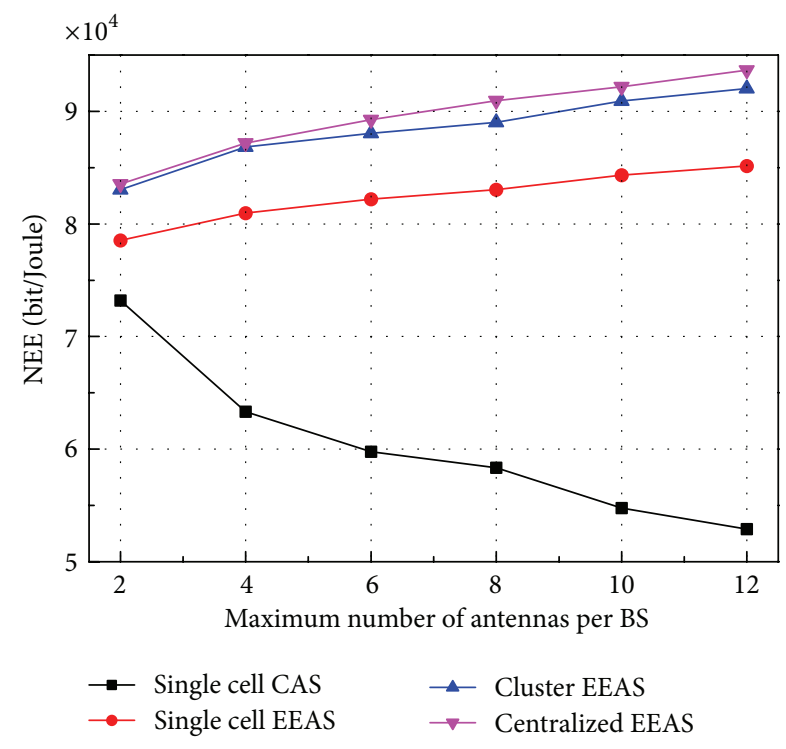

FIGURE 4: NEE versus maximum number of antennas per BS with $P=20 \mathrm{~W}, 10$ users per cell, and cell radius of $1 \mathrm{Km}$.

transmit and receive antenna sets cooperatively. And it needs complete CSI sharing that will bring large amount of signaling overhead. Furthermore, the four algorithms have the same computation complexity.

\section{Conclusion}

This paper analyzes the available network energy efficiency through transmit and receive antenna selection for multicell MU-MIMO system. In this paper, we first deduce an ergodic energy efficiency based on large scale CSI. Based on this energy efficiency, we introduce a cost function of transmit and receive antenna selection and convert the transmit and receive antenna selection problem to local optimization problem based on the maximization of BS cluster energy efficiency. Then, we propose a antenna selection scheme based on cluster energy efficiency (cluster EEAS). The simulation results show that the proposed scheme performs better network energy efficiency compared to the single cell CAS and single cell EEAS and can achieve about 98\% NEE of the centralized EEAS. Besides, since the proposed scheme uses large scale CSI to implement antenna selection and do not need the complete CSI of neighbor cells' users, it can effectively reduce the signaling overhead brought by the exchange of CSI.

\section{Appendix}

When $J=3$, (12) can be expressed as

$$
X_{k, i}=\frac{\alpha_{k, i} Z_{k, i}}{1+\beta_{k, 1} Y_{k, 1}+\beta_{k, 2} Y_{k, 2}+\beta_{k, 3} Y_{k, 3}} .
$$

In order to obtain the distribution function of $X_{k, i}$, first we need to obtain the probability density function of $\beta_{k, 1} Y_{k, 1}+$ $\beta_{k, 2} Y_{k, 2}+\beta_{k, 3} Y_{k, 3}$. For the sake of convenience in writing, we omit the subscript in (A.1), then (A.1) can be rewritten as

$$
X=\frac{\alpha Z}{1+\beta_{1} Y_{1}+\beta_{2} Y_{2}+\beta_{3} Y_{3}}
$$

The probability density function of Chi-square distribution with degree of freedom $2 M$ is

$$
f(x)=\frac{x^{M-1} e^{-x / r_{1}}}{(M-1) ! r_{1}^{M}} .
$$

The probability density function of Chi-square distribution with degree of freedom 2 is,

$$
f(x)=\frac{e^{-x / r_{1}}}{r_{1}} .
$$

Define $\bar{Y}=\beta_{1} Y_{1}+\beta_{2} Y_{2}$, and $Y_{1}$ and $Y_{2}$ are mutually independent.

One has

$$
\begin{aligned}
f(\bar{y}) & =\int_{-\infty}^{+\infty} f(y) f(\bar{y}-y) d y \\
& =\int_{0}^{\bar{y}} \frac{e^{-y / \beta_{1}}}{\beta_{1}} \frac{e^{-(\bar{y}-y) / \beta_{2}}}{\beta_{2}} d y \\
& =\frac{1}{\beta_{1} \beta_{2}} \int_{0}^{\bar{y}} e^{-y / \beta_{1}} e^{-(\bar{y}-y) / \beta_{2}} d y \\
& =\frac{1}{\beta_{1} \beta_{2}} e^{-\bar{y} / \beta_{2}} \int_{0}^{\bar{y}} e^{-y / \beta_{1}} e^{y / \beta_{2}} d y .
\end{aligned}
$$

Define $y / \beta_{1}-y / \beta_{2}=z$, then $f(\bar{y})$ can be rewritten as

$$
\begin{aligned}
f(\bar{y}) & =-\frac{1}{\beta_{1}-\beta_{2}} e^{-\bar{y} / \beta_{2}} \int_{0}^{\left(\left(\beta_{2}-\beta_{1}\right) / \beta_{1} \beta_{2}\right) \bar{y}} e^{-z} d z \\
& =\frac{1}{\beta_{1}-\beta_{2}} e^{-\bar{y} / \beta_{2}} e^{-z} \mid \begin{array}{c}
\frac{\beta_{2}-\beta_{1}}{\beta_{1} \beta_{2}} \bar{y} \\
0
\end{array} \\
& =\frac{1}{\beta_{1}-\beta_{2}}\left(e^{-\bar{y} / \beta_{1}}-e^{-\bar{y} / \beta_{2}}\right) .
\end{aligned}
$$

Define $Y=\bar{Y}+\beta_{3} Y_{3}$, and $\bar{Y}$ and $Y_{3}$ are mutually independent. 
One has

$$
\begin{aligned}
& f(y)=\int_{-\infty}^{+\infty} f(\bar{y}) f(y-\bar{y}) d \bar{y} \\
& =\int_{0}^{x} \frac{1}{\beta_{1}-\beta_{2}}\left(e^{-\bar{y} / \beta_{1}}-e^{-\bar{y} / \beta_{2}}\right) \frac{e^{-(x-\bar{y}) / \beta_{3}}}{\beta_{3}} d \bar{y} \\
& =\frac{1}{\left(\beta_{1}-\beta_{2}\right) \beta_{3}} e^{-y / \beta_{3}} \\
& \times\left[\int_{0}^{y} e^{-\bar{y} / \beta_{1}+\bar{y} / \beta_{3}} d \bar{y}-\int_{0}^{y} e^{-\bar{y} / \beta_{2}+\bar{y} / \beta_{3}} d \bar{y}\right] \\
& =\frac{1}{\left(\beta_{1}-\beta_{2}\right) \beta_{3}} e^{-y / \beta_{3}} \\
& \times\left[\frac{\beta_{1} \beta_{3}}{\beta_{3}-\beta_{1}} \int_{0}^{\left(\left(\beta_{3}-\beta_{1}\right) / \beta_{1} \beta_{3}\right) y} e^{-z} d z\right. \\
& \left.-\frac{\beta_{2} \beta_{3}}{\beta_{3}-\beta_{2}} \int_{0}^{\left(\left(\beta_{3}-\beta_{2}\right) / \beta_{2} \beta_{3}\right) y} e^{-z} d z\right] \\
& =\frac{1}{\left(\beta_{1}-\beta_{2}\right) \beta_{3}} e^{-y / \beta_{3}} \\
& \times\left[\frac{\beta_{1} \beta_{3}}{\beta_{1}-\beta_{3}}\left(e^{-\left(\left(\beta_{3}-\beta_{1}\right) / \beta_{1} \beta_{3}\right) y}-1\right)\right. \\
& \left.-\frac{\beta_{2} \beta_{3}}{\beta_{2}-\beta_{3}}\left(e^{-\left(\left(\beta_{3}-\beta_{2}\right) / \beta_{2} \beta_{3}\right) y}-1\right)\right] \\
& =\frac{\beta_{1}}{\left(\beta_{1}-\beta_{2}\right)\left(\beta_{1}-\beta_{3}\right)}\left(e^{-y / \beta_{1}}-e^{-y / \beta_{3}}\right) \\
& -\frac{\beta_{2}}{\left(\beta_{1}-\beta_{2}\right)\left(\beta_{2}-\beta_{3}\right)}\left(e^{-y / \beta_{2}}-e^{-y / \beta_{3}}\right) \\
& =\frac{\beta_{1}}{\left(\beta_{1}-\beta_{2}\right)\left(\beta_{1}-\beta_{3}\right)}\left(e^{-y / \beta_{1}}-e^{-y / \beta_{3}}\right) \\
& -\frac{\beta_{2}}{\left(\beta_{1}-\beta_{2}\right)\left(\beta_{2}-\beta_{3}\right)}\left(e^{-y / \beta_{2}}-e^{-y / \beta_{3}}\right) \\
& =\frac{\beta_{1}}{\left(\beta_{1}-\beta_{2}\right)\left(\beta_{1}-\beta_{3}\right)} e^{-y / \beta_{1}} \\
& -\frac{\beta_{2}}{\left(\beta_{1}-\beta_{2}\right)\left(\beta_{2}-\beta_{3}\right)} e^{-y / \beta_{2}} \\
& +\frac{\beta_{3}}{\left(\beta_{1}-\beta_{3}\right)\left(\beta_{2}-\beta_{3}\right)} e^{-y / \beta_{3}} .
\end{aligned}
$$

Now, we have already obtained the probability density function of $\beta_{k, 1} Y_{k, 1}+\beta_{k, 2} Y_{k, 2}+\beta_{k, 3} Y_{k, 3}$, and then $X$ can be expressed as

$$
X=\frac{\alpha Z}{1+Y} .
$$

From the form of the probability density function of $\beta_{k, 1} Y_{k, 1}+$ $\beta_{k, 2} Y_{k, 2}+\beta_{k, 3} Y_{k, 3}$, we can see that its PDF is a linear combination of three PDFs which obey Chi-square distribution, and each part of that linear combination is a product of a Chisquare distribution and a constant; that is,

$$
\begin{aligned}
f(y)= & \frac{\beta_{1}^{2}}{\left(\beta_{1}-\beta_{2}\right)\left(\beta_{1}-\beta_{3}\right)} \frac{e^{-y / \beta_{1}}}{\beta_{1}} \\
& -\frac{\beta_{2}^{2}}{\left(\beta_{1}-\beta_{2}\right)\left(\beta_{2}-\beta_{3}\right)} \frac{e^{-y / \beta_{2}}}{\beta_{2}} \\
& +\frac{\beta_{3}^{2}}{\left(\beta_{1}-\beta_{3}\right)\left(\beta_{2}-\beta_{3}\right)} \frac{e^{-y / \beta_{3}}}{\beta_{3}} .
\end{aligned}
$$

Assume that $Z$ obeys Chi-square distribution with degree of freedom $2 M$. When $J=1$, the distribution function of $X$ is $\bar{F}_{X}(x)=1-\sum_{r=0}^{M-1} \sum_{l=0}^{r}\left(\alpha^{l+1-r} / \beta(r-\right.$ $l) !\left(x^{r} e^{-x / \alpha} /(x+\alpha / \beta)^{l+1}\right)$. When $J=3$, the distribution function of $X, F_{X}(x)$, can be expressed as

$$
\begin{aligned}
& F_{X}(x)=\frac{\beta_{1}^{2}}{\left(\beta_{1}-\beta_{2}\right)\left(\beta_{1}-\beta_{3}\right)} \\
& \times\left(1-\sum_{r=0}^{M-1} \sum_{l=0}^{r} \frac{\alpha^{l+1-r}}{\beta_{1}(r-l) !} \frac{x^{r} e^{-x / \alpha}}{\left(x+\alpha / \beta_{1}\right)^{l+1}}\right) \\
& -\frac{\beta_{2}^{2}}{\left(\beta_{1}-\beta_{2}\right)\left(\beta_{2}-\beta_{3}\right)} \\
& \times\left(1-\sum_{r=0}^{M-1} \sum_{l=0}^{r} \frac{\alpha^{l+1-r}}{\beta_{2}(r-l) !} \frac{x^{r} e^{-x / \alpha}}{\left(x+\alpha / \beta_{2}\right)^{l+1}}\right) \\
& +\frac{\beta_{3}^{2}}{\left(\beta_{1}-\beta_{3}\right)\left(\beta_{2}-\beta_{3}\right)} \\
& \times\left(1-\sum_{r=0}^{M-1} \sum_{l=0}^{r} \frac{\alpha^{l+1-r}}{\beta_{3}(r-l) !} \frac{x^{r} e^{-x / \alpha}}{\left(x+\alpha / \beta_{3}\right)^{l+1}}\right) \\
& =1-\frac{\beta_{1}}{\left(\beta_{1}-\beta_{2}\right)\left(\beta_{1}-\beta_{3}\right)} \\
& \times \sum_{r=0}^{M-1} \sum_{l=0}^{r} \frac{\alpha^{l+1-r}}{(r-l) !} \frac{x^{r} e^{-x / \alpha}}{\left(x+\alpha / \beta_{1}\right)^{l+1}} \\
& -\frac{\beta_{2}}{\left(\beta_{1}-\beta_{2}\right)\left(\beta_{2}-\beta_{3}\right)} \\
& \times \sum_{r=0}^{M-1} \sum_{l=0}^{r} \frac{\alpha^{l+1-r}}{(r-l) !} \frac{x^{r} e^{-x / \alpha}}{\left(x+\alpha / \beta_{2}\right)^{l+1}} \\
& +\frac{\beta_{3}}{\left(\beta_{1}-\beta_{3}\right)\left(\beta_{2}-\beta_{3}\right)} \\
& \times \sum_{r=0}^{M-1} \sum_{l=0}^{r} \frac{\alpha^{l+1-r}}{(r-l) !} \frac{x^{r} e^{-x / \alpha}}{\left(x+\alpha / \beta_{3}\right)^{l+1}} .
\end{aligned}
$$


Thus, we can obtain $E[\ln (1+X)]$ for $J=3$ as follows:

$$
\begin{aligned}
& E[\ln (1+X)] \\
& =\sum_{r=0}^{M-1} \sum_{l=0}^{r} \frac{\left(\alpha_{k, i}\right)^{l+1-r}}{(r-l) !} \\
& \times\left[\begin{array}{c}
\frac{\beta_{1}}{\left(\beta_{1}-\beta_{2}\right)\left(\beta_{1}-\beta_{3}\right)} I_{1}\left(\frac{1}{\alpha}, \frac{\alpha}{\beta_{1}}, r, l+1\right) \\
-\frac{\beta_{2}}{\left(\beta_{1}-\beta_{2}\right)\left(\beta_{2}-\beta_{3}\right)} I_{1}\left(\frac{1}{\alpha}, \frac{\alpha}{\beta_{2}}, r, l+1\right) \\
+\frac{\beta_{3}}{\left(\beta_{1}-\beta_{3}\right)\left(\beta_{2}-\beta_{3}\right)} I_{1}\left(\frac{1}{\alpha}, \frac{\alpha}{\beta_{3}}, r, l+1\right)
\end{array}\right],
\end{aligned}
$$

where $I_{1}(a, b, m, n)=\int_{0}^{\infty}\left(x^{m} e^{-a x} /(x+b)^{n}(x+1)\right) d x$.

The close-form solution of this integral is in [31].

\section{Acknowledgment}

This work was supported by the National S\&T Major Project (2012ZX03003-001).

\section{References}

[1] 3GPP TR 32.826, Telecommunication management; Study on Energy Savings Management (ESM), (Release 10), 2010, http://www.3gpp.org.

[2] Z. Hasan, H. Boostanimehr, and V. K. Bhargava, "Green cellular networks: a survey, some research issues and challenges," IEEE Communications Surveys and Tutorials, vol. 13, no. 4, pp. 524540, 2011.

[3] G. Miao, N. Himayat, and G. Y. Li, "Energy-efficient link adaptation in frequency-selective channels," IEEE Transactions on Communications, vol. 58, no. 2, pp. 545-554, 2010.

[4] "Further Advancements for E-UTRA Physical Layer Aspects," Tech. Rep. TR 36.814 V9.0.0 (Release 9), 2010, http://www.3gpp.org.

[5] M. Peng and W. Wang, "Technologies and standards for TDSCDMA evolutions to IMT-advanced," IEEE Communications Magazine, vol. 47, no. 12, pp. 50-58, 2009.

[6] M. Peng, W. Wang, and H. H. Chen, "TD-SCDMA evolution," IEEE Vehicular Technology Magazine, vol. 5, no. 2, pp. 28-41, 2010.

[7] C. Han and S. Armour, "Energy efficient radio resource management strategies for green radio," IET Communication, vol. 5, no. 18, pp. 2629-2639, 2011.

[8] M. Peng, X. Zhang, W. Wang, and H. Chen, "Performance of dual-polarized MIMO for TD-HSPA evolution systems," IEEE Systems Journal, vol. 5, no. 3, pp. 406-416, 2011.

[9] A. Molisch and M. Z. Win, "MIMO systems with antenna selection," IEEE Microwave Magazine, vol. 5, no. 1, pp. 46-56, 2004.

[10] A. Molisch, M. Z. Win, Y.-S. Choi, and J. H. Winters, "Capacity of MIMO systems with antenna selection," IEEE Transactions on Wireless Communications, vol. 4, no. 4, pp. 1759-1772, 2005.

[11] R. Chen, Z. Shen, J. G. Andrews, and R. W. Heath, "Multimode transmission for multiuser MIMO systems with block diagonalization," IEEE Transactions on Signal Processing, vol. 56, no. 7, pp. 3294-3302, 2008.
[12] M. Gharavi-Alkhansari and A. B. Gershman, "Fast antenna subset selection in MIMO systems," IEEE Transactions on Signal Processing, vol. 52, no. 2, pp. 339-347, 2004.

[13] Z. Shen, R. Chen, J. G. Andrews, R. W. Heath, and B. L. Evans, "Low complexity user selection algorithms for multiuser MIMO systems with block diagonalization," IEEE Transactions on Signal Processing, vol. 54, no. 9, pp. 3658-3663, 2006.

[14] Y. Liu, Y. Zhang, C. Ji, W. Q. Malik, and D. J. Edwards, "A lowcomplexity receive-antenna-selection algorithm for MIMOOFDM wireless systems," IEEE Transactions on Vehicular Technology, vol. 58, no. 6, pp. 2793-2802, 2009.

[15] L. Sun and M. R. McKay, "Eigen-based transceivers for the MIMO broadcast channel with semi-orthogonal user selection," IEEE Transactions on Signal Processing, vol. 58, no. 10, pp. 52465261, 2010.

[16] J. K. Lain, "Joint transmit/receive antenna selection for MIMO systems: a real-valued genetic approach," IEEE Communications Letters, vol. 15, no. 1, pp. 58-60, 2011.

[17] T. Gucluoglu and T. M. Duman, "Performance analysis of transmit and receive antenna selection over flat fading channels," IEEE Transactions on Wireless Communications, vol. 7, no. 8, pp. 3056-3065, 2008.

[18] J. W. Kang, H. W. Je, C. S. Park, and K. B. Lee, “Transmit antenna subset selection for downlink MIMO systems in multicell environments," IEEE Transactions on Wireless Communications, vol. 9, no. 7, pp. 2113-2118, 2010.

[19] T. Zhou, M. Peng, W. Wang, and H. Chen, "Lowcomplexity coordinated beamforming for downlink multicell SDMA/OFDM system," IEEE Transactions Vehicular Technology, vol. 62, no. 1, pp. 247-255, 2013.

[20] S. Cui, A. J. Goldsmith, and A. Bahai, "Energy-efficiency of MIMO and cooperative MIMO techniques in sensor networks," IEEE Journal on Selected Areas in Communications, vol. 22, no. 6, pp. 1089-1098, 2004.

[21] M. T. Kakitani, G. Brante, R. D. Souza, and M. A. Imran, "Energy efficiency of transmit diversity systems under a realistic power consumption model," IEEE Communications Letters, vol. 17, no. 1, pp. 119-122, 2013.

[22] J. Joung, C. K. Ho, and S. Sun, "Power amplifier switching (PAS) for energy efficient systems," IEEE Wireless Communications Letters, vol. 2, no. 1, pp. 14-17, 2013.

[23] C. Jiang and L. J. Cimini, "Antenna selection for energy efficient MIMO transmission," IEEE Wireless Communication Letters, vol. 1, no. 6, pp. 577-580, 2012.

[24] J. Xu and L. Qiu, "Energy efficiency optimization for MIMO broadcast channels," IEEE Transactions Wireless Communication, vol. 12, no. 2, pp. 690-701, 2013.

[25] H. Li, G. P. Koudouridis, and J. Zhang, "Antenna selection schemes for energy efficiency in distributed antenna systems," in Proceedings of the IEEE International Conference on Communications (ICC '12), pp. 5619-5623, 2012.

[26] D. W. K. Ng, E. S. Lo, and R. Schober, "Energy-efficient resource allocation in SDMA systems with large numbers of base station antennas," in Proceedings of the IEEE International Conference on Communications (ICC '12), pp. 4027-4032, 2012.

[27] C. Jiang and L. J. Cimini, "Antenna selection for energy-efficient MIMO transmission," IEEE Wireless Communications Letters, vol. 1, no. 6, pp. 577-580, 2012.

[28] G. Lim and L. J. Cimini Jr., "Energy-efficient cooperative beamforming in clustered wireless networks," IEEE Transactions on Wireless Communications, vol. 12, no. 3, pp. 1376-1385, 2013. 
[29] S. Li and L. Qiu, "Improving network energy efficiency through transmit antenna number and transmission mode selection in multicell systems," in IEEE Vehicular Technology Conference (VTC '12), pp. 1-5, 2012.

[30] Q. H. Spencer, A. L. Swindlehurst, and M. Haardt, "Zero-forcing methods for downlink spatial multiplexing in multiuser MIMO channels," IEEE Transactions on Signal Processing, vol. 52, no. 2, pp. 461-471, 2004.

[31] J. Zhang and J. G. Andrews, "Adaptive spatial intercell interference cancellation in multicell wireless networks," IEEE Journal on Selected Areas in Communications, vol. 28, no. 9, pp. 1455$1468,2010$. 

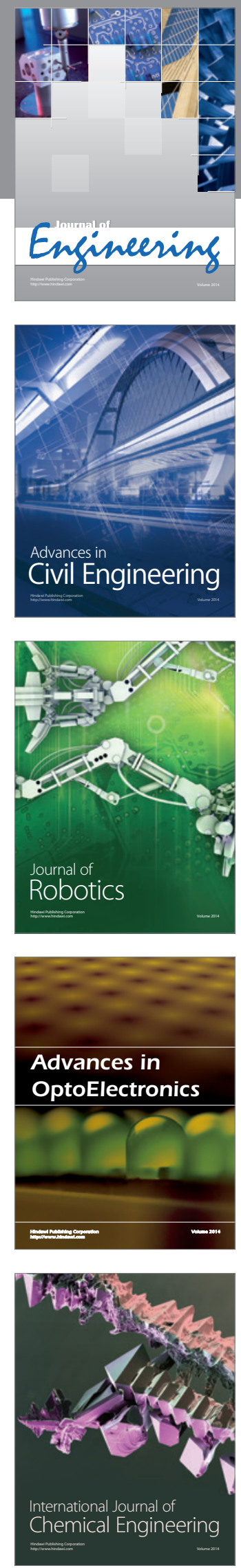

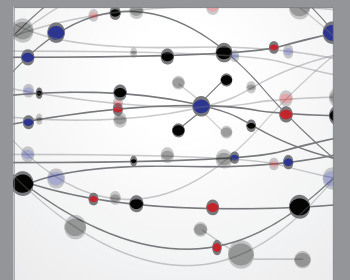

The Scientific World Journal
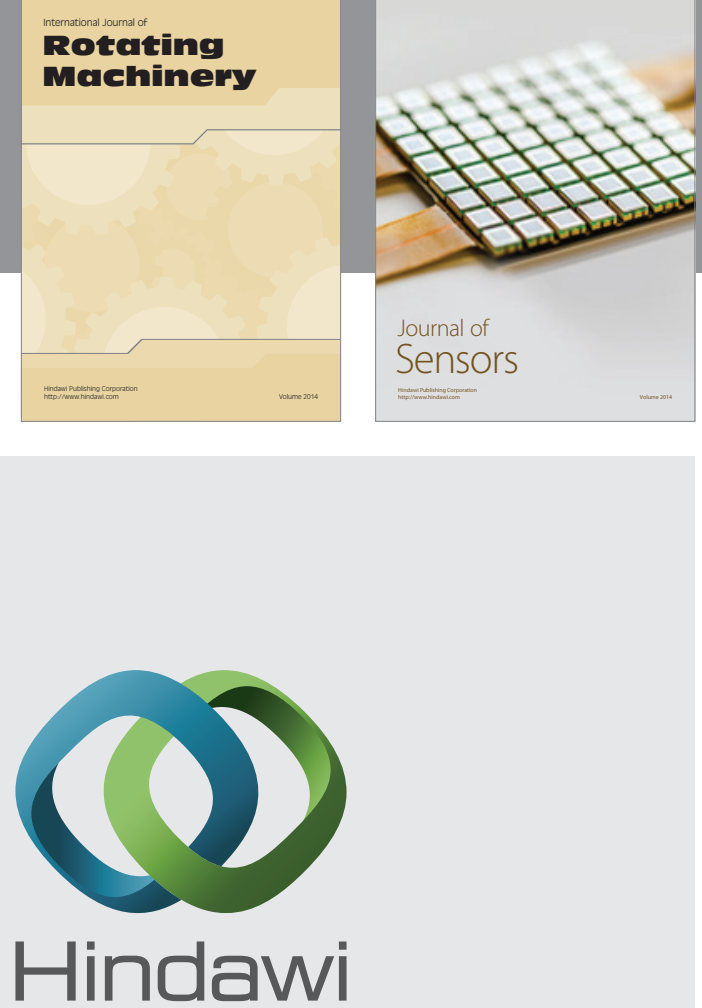

Submit your manuscripts at http://www.hindawi.com
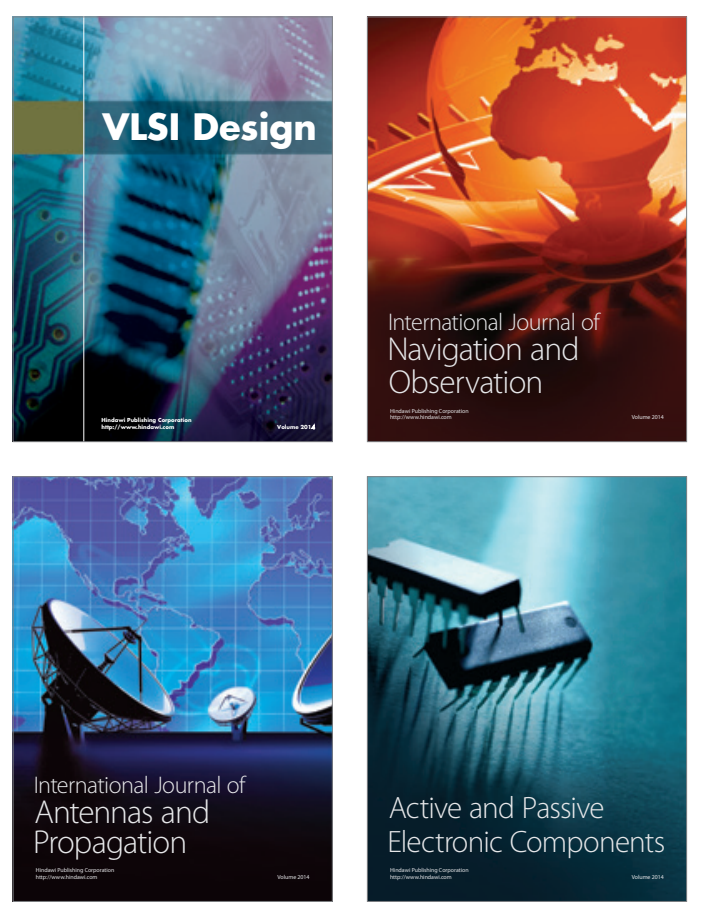
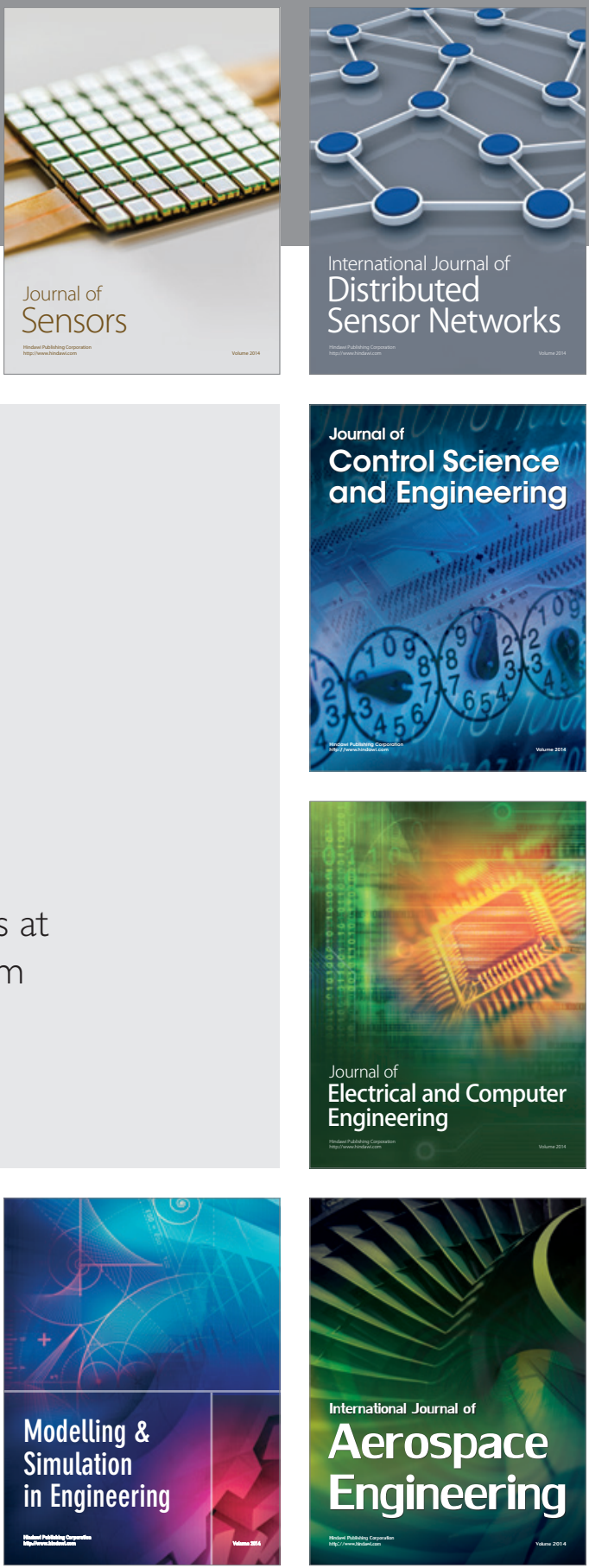

Journal of

Control Science

and Engineering
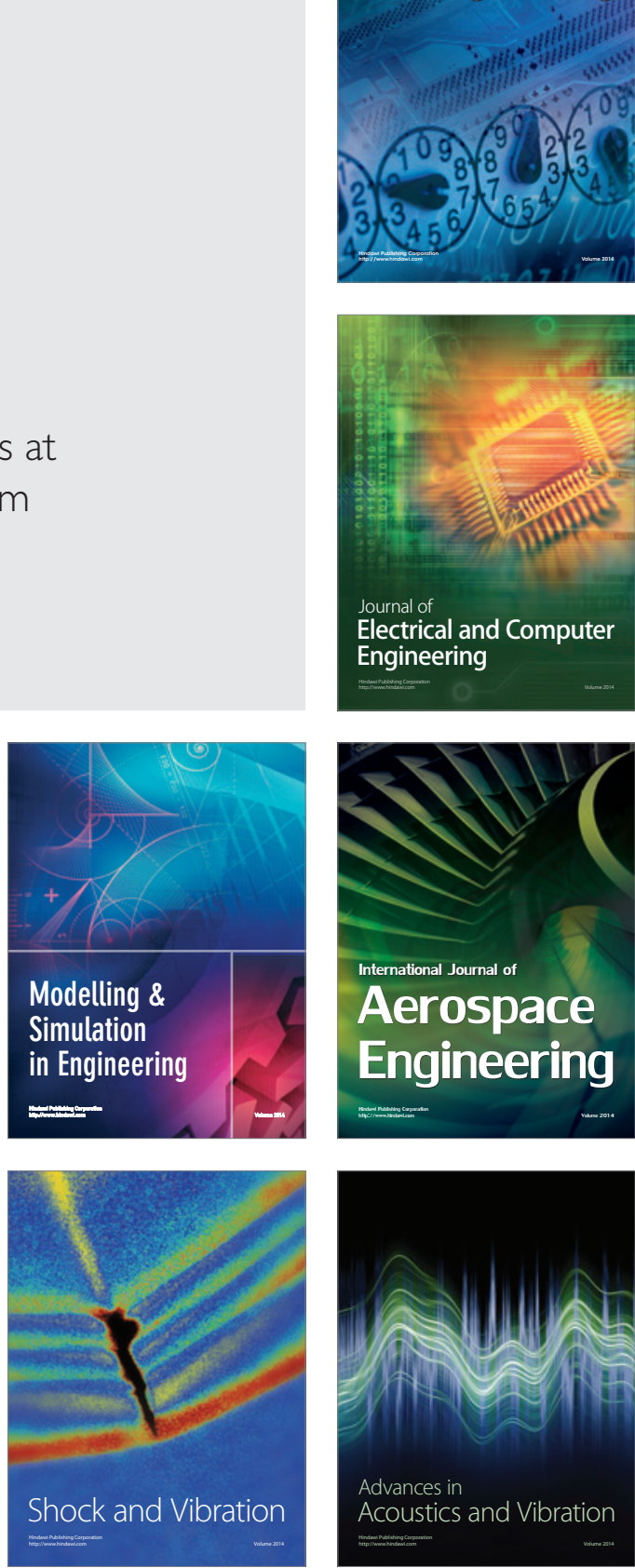